\title{
Demanda por Ensino Superior: o caso da Universidade Federal da Bahia
}

\author{
CLÁUDIO PONDÉ AVENA ${ }^{1}$ \\ Doutorando em Educação (UFBa/FACED) \\ Mestre em Economia (UFBa/CME) \\ Professor da Faculdade Ruy Barbosa \\ cpavena@ufba.br
}

\begin{abstract}
Resumo
Este artigo apresenta uma metodologia inovadora para estimação da demanda por educação. Além disso, ele estima, em seção transversal, as demandas agregadas pelos cursos existentes na UFBa a partir da base de dados do Vestibular para os anos de 1993 a 2001(exceto os anos de 1996, 1999 e 2000). Para isto, desenvolvem-se modelos de estimação do custo de oportunidade e de renda vitalícia do aluno. Esta última pode ser interpretada como o estoque de capital humano do indivíduo com educação superior na Região Metropolitana de Salvador. Os modelos estimados apresentam um poder de explicação excepcionalmente alto, com especial destaque para a renda vitalícia, que explica mais de $80 \%$ da variabilidade da demanda. Isto demonstra a viabilidade de se utilizar o número de matrículas como proxy para o estoque de capital humano em modelos de crescimento econômico, como é usual na literatura de growth accounting. Outros resultados são apresentados, como as elasticidades da demanda e a taxa marginal de substituição entre o custo de oportunidade e a renda vitalícia.

Palavras-chave: economia da educação, educação e trabalho, demanda por ensino superior, capital humano.
\end{abstract}

\section{Resumen}

Este trabajo presenta una metodología innovadora para la estimación de la demanda por la educación. Además, a partir de datos transversales de la base de la Selectividad de los años de 1993 a 2001 (con excepción de los años 1996, 1999 y 2001), este trabajo estima la demanda agregada por los estudios ofrecidos por la UFBa. Para eso, se desarrollan modelos de estimativa del coste de oportunidad y de la renta vitalicia del alumno. Esta última puede ser entendida como la cantidad de capital humano existente en la Región Metropolitana de Salvador. Los modelos estimados presentan un alto poder de explicación, especialmente sobre la renta vitalicia, que responde por más del $80 \%$ de la variabilidad de la demanda.

1 Desejo agradecer ao meu orientador, Prof. Dr. Robert E. Verhine, por ter possibilitado o meu acesso à base de dados do Vestibular da UFBa e pelo incentivo para que realizasse este Doutorado; aos funcionários da UFBa/CPD, Antônio Ribeiro e Cora Maria, pela boa vontade com que atenderam à minha demanda por estes dados, e à Faculdade Ruy Barbosa pelo apoio institucional, o que tem possibilitado esta e outras pesquisas. Como de praxe, eventuais erros neste trabalho são de minha inteira responsabilidade. Comentários e sugestões sobre este artigo são bem vindos. Favor enviá-los para o endereço eletrônico. 
Esto demuestra la viabilidad de utilizar el número de matrículas como proxy para la cantidad de capital humano en modelos de crecimiento económico, como es usual en la literatura sobre growth accounting. Otros resultados obtenidos se refieren a la elasticidad de la demanda y a la tasa marginal de substitución entre el coste de oportunidad y la renta vitalicia.

Palabras-clave: economía de la educación, educación y trabajo, demanda de enseñanza superior, capital humano.

\begin{abstract}
This paper presents an innovative methodology for estimating demand for education. In addition, it estimates, with cross-sectional data, the aggregate demand for the fields of study offered at UFBa. The data used are from the Vestibular (entrance examination) database for the years 1993 through 2001 (except for the years 1996, 1999 and 2000). The study develops estimation models for one's forgone and lifetime earnings. The latter can be understood as the stock of higher educated human capital in the Metropolitan Region of Salvador. The statistical models show a very high explanation power, especially those for the lifetime earnings which account for over $80 \%$ of the variability of the demand. This demonstrates that enrollments are a good proxy for human capital stock, as is generally assumed in the growth accounting literature. Moreover, the paper estimates the elasticities of demand and the marginal rate of substitution between forgone and lifetime earnings.

Key words: economics of education, education and work, demand for higher education, human capital.
\end{abstract}




\section{INTRODUÇÃO}

É lugar comum na literatura reconhecer amplamente a importância da educação para o desenvolvimento econômico, para a melhoria na distribuição de renda e para o desenvolvimento tecnológico de um país. Freeman (1995), estudioso da economia da tecnologia, mostrou a importância da educação de nível superior como um componente essencial do sistema nacional de inovação para o rápido crescimento de um país, como foi o caso da Coréia do Sul. Além disso, a educação superior é cada vez mais importante para que a inserção do indivíduo no mercado de trabalho seja mais provável (aumento da empregabilidade) e os rendimentos, em média, sejam bem mais elevados. Este é o caso da Região Metropolitana de Salvador (Avena, 2000).

Nos últimos anos, muitas têm sido as faculdades que iniciaram as suas atividades na Região Metropolitana de Salvador, ampliando-se, sobremaneira, a oferta de vagas em diversas profissões, com particular destaque para o curso de Administração de Empresas. As instituições públicas de ensino superior, entretanto, não são muitas nesta região, resumindo-se à Universidade do Estado da Bahia (UNEB) e à Universidade Federal da Bahia (UFBa.). Discute-se atualmente, no âmbito da UFBa, a criação de mais uma instituição federal de ensino superior, que se chamaria Universidade do Recôncavo, no Campus de Cruz das Almas.

Será que haverá demanda por esses cursos? Quais os fatores mais importantes na decisão de se ingressar na educação superior? Como se dá a escolha da profissão por parte do aluno? Deveria haver cotas para negros e/ou para mulheres? Neste artigo procurar-se-á responder às três primeiras questões e lançar luzes sobre a última. Como se trata de um estudo preliminar, pretendemos responder a essas e a outras perguntas por meio de uma modelagem alternativa em um outro trabalho ${ }^{2}$. Entenda-se por modelagem alternativa a utilização de regressão logística. Utilizaram-se dessa técnica Desjardins et al. (1999), Hagy e Staniec (2002) e Montmarquette (2002), assim como os inúmeros trabalhos revisados por Hossler et al. (1989).

Conhecer a demanda por educação em uma instituição de ensino superior, seja ela pública e gratuita, ou particular, fornece sempre informações valiosas para as estratégias de seleção (tornando-a mais justa, eqüitativa e democrática), mercadológicas (no caso de instituições particulares) e para se adequar a oferta de vagas à demanda, de modo a

2 Este artigo faz parte da pesquisa de Doutorado do autor. 
fazer um uso mais racional dos recursos escassos, sejam estes públicos ou não.

Se os recursos forem públicos, razão ainda maior para não se descuidar da otimização dos mesmos, haja vista a existência de imensas necessidades básicas da população brasileira por serem ainda atendidas, da crise fiscal do Estado brasileiro e das universidades federais; alguns números dão a dimensão da importância desses gastos com o ensino superior, a exemplo dos 3,9\% dos gastos públicos totais que são destinados para esta finalidade, sendo que $95 \%$ deles são com salários, segundo estimativas realizadas para o ano de 1997. (Verhine, 2002). Além disto, anualmente, $50,5 \%, 46,5 \%$ e $44,1 \%$ dos alunos ingressos (aprovados no Vestibular), concluíram o curso nos anos de 1993, 1994 e 1995, respectivamente, o que representa uma grande ociosidade de recursos. As principais razões para esses índices de conclusão são recusa de matrícula, cancelamento de vaga, reprovação ou desistência do curso. Selecionar, portanto, candidatos com uma maior probabilidade de conclusão do curso é algo de grande relevância econômica e social na medida em que se poderá elevar o índice de concluintes, evitando-se grande desperdício de recursos.

O candidato ao concurso Vestibular da Universidade Federal da Bahia (UFBa) se depara com a necessidade de escolher um dentre os 51 cursos oferecidos. Estes 51 cursos constituem um conjunto de escolhas possíveis. Esta é uma escolha que irá definir a profissão e a carreira do candidato, provavelmente, para o resto de sua vida. Às vezes ocorre de o candidato mudar de curso, mas isto tende a reduzir-se à medida que o indivíduo avança no curso, pois os custos incorridos relativos à renda sacrificada do aluno tendem a se transformar em custos irrecuperáveis, principalmente se a nova escolha implicar o aproveitamento de poucos créditos na nova escolha; assim, em caso de mudança, os custos irrecuperáveis são tão maiores quanto menor a complementaridade entre os cursos.

Neste artigo, considera-se que cada curso é um bem ${ }^{3}$ e a escolha dos candidatos parte da hipótese de maximização de sua função utilidade, dada a sua restrição orçamentária vitalícia. Desenvolvem-se modelos de estimação de demanda educacional de forma inovadora, utilizando-se da teoria de demanda agregada clássica. $\mathrm{Na}$ maior parte da literatura mencionada anteriormente, faz-se uso de modelos de demanda a partir de

3 Há um debate na literatura da teoria do capital humano se a educação é um bem de consumo ou de investimento. Neste artigo considerou-se que seja um bem de investimento, daí porque se trabalhou com a renda vitalícia. Entretanto, para efeito de demanda, não se parece que esta disputa seja importante. 
estimações por meio de regressões logísticas ${ }^{4}$, tal como foi inaugurado por McFadden (1973).

No modelo de demanda aqui desenvolvido, apoiando-se em MasColell et al.(1995) e Varian (1984), os candidatos maximizarão uma função utilidade $\mathrm{U}=\mathrm{U}\left(\mathrm{c}_{1}, \mathrm{c}_{2}, \ldots, \mathrm{c}_{\mathrm{n}}\right)$, onde $\mathrm{n}$ é o número total de cursos que o indivíduo se depara na sua escolha. A priori, a decisão do indivíduo não depende apenas de sua renda vitalícia e de seu custo de oportunidade de escolher um curso e deixar de perceber um salário relativo à sua qualificação de ensino médio. Outras variáveis tais como o sexo, o nível de escolaridade dos pais, a ocupação dos pais (o candidato pode ser influenciado pela profissão dos pais), a etnia do candidato, se o candidato é da capital ou interior, se ele é proveniente de escola particular ou pública, se é solteiro ou não, número de vezes que prestou concurso vestibular e expectativa em relação ao curso podem influenciar nessa decisão.

O objetivo deste artigo é verificar se, e em que medida, essas variáveis afetam a escolha do curso que realizará. Este artigo se estrutura da seguinte maneira: além desta introdução, na segunda seção desenvolvese o modelo de demanda a ser utilizado e os modelos para cálculo de renda vitalícia e do custo de oportunidade do aluno; na terceira seção discutem-se as duas bases de dados utilizadas; na quarta seção, apresentam-se os modelos econométricos, discutem-se os resultados encontrados e faz-se um exercício de estática comparativa; e, por fim, se apresentam as principais conclusões.

\section{DESENVOLVIMENTO DO MODELO TEÓRICO}

Uma vez que o candidato i demanda uma única vaga no curso $\mathrm{j}$ da Universidade, se trata de uma demanda descontínua definida por:

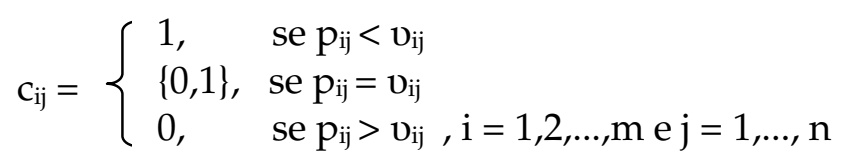

No caso em apreço, como há uma grande quantidade de candidatos inscritos, as preferências individuais apresentam uma dispersão em torno do preço $p_{i j}=v_{i j}$, onde $\mathrm{v}_{\mathrm{ij}}$ é a variável aleatória contínua com distribuição de probabilidade, $\mathrm{G}\left(\mathrm{v}_{\mathrm{ij}}\right)$, e representa o preço de reserva do candidato.

4 Regressão logística é muito utilizada quando a variável dependente é binária, do tipo, 0 ou 1. 
Fazendo-se $\Sigma_{\mathrm{i}=1, \ldots, \mathrm{m}} \mathrm{c}_{\mathrm{ij}}=\mathrm{c}_{\mathrm{j}}$, onde $\mathrm{c}_{\mathrm{j}}$ é a demanda agregada (número total de candidatos inscritos) pelo curso j, que é contínua, apesar de que a demanda de cada candidato não o ser. (Mas-Collel et al., 1995; Varian, 1984).

$c_{j}=c_{j}\left(\mathbf{p}, \Sigma_{i=1, \ldots, m}, R_{1 j}\right)$ é a função de demanda agregada por cada curso

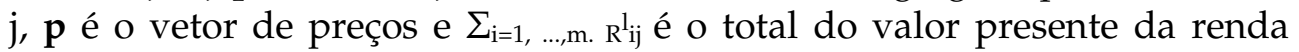
vitalícia de todos os candidatos inscritos no Vestibular, no curso j.

Sendo o conjunto orçamentário $B_{p, R}=\left\{c_{j} \in R_{+}{ }^{n+p}:\right.$ p.c $\left.\leq R^{1}\right\}$ compacto, a função objetivo tem um máximo dado pelo problema de maximização condicionada definido pelo sistema (2).

$$
\begin{aligned}
& \underset{c_{j}}{\operatorname{Max} U\left(c_{1}, c_{2}, \ldots, c_{n}, c_{n+1}, \ldots, c_{n+p}\right)} \\
& \text { s.a. } \Sigma_{j=1, \ldots, n+p} p_{j} . c_{j} \leq R_{j}^{1} \text { e } c_{j}>0, \text { para todo } j .
\end{aligned}
$$

Onde,

U( . ) é a função de utilidade dos candidatos, diferenciável em todo o seu domínio e quase côncava;

$\mathrm{c}_{\mathrm{j}}(\mathrm{j}=1,2, \ldots, \mathrm{n})$ é a quantidade demandada anual pelo curso $\mathrm{j} ;$

$\mathrm{p}_{\mathrm{j}}$ é o preço do bem j; no caso de interesse, é o valor presente do custo de oportunidade médio (descontado à taxa de juro instantânea $\beta$ ) dentre os candidatos ao curso $j$; ou seja $\mathrm{p}_{\mathrm{j}}=\left(\sum_{\mathrm{i}=1, \ldots, \mathrm{m}} \mathrm{p}_{\mathrm{ij}}\right) / \mathrm{m}$, é a média dos custos de oportunidade dentre os $\mathrm{m}$ candidatos ao curso j, calculado por meio de análise de regressão, para que se controlem vários efeitos que afetam esse custo. Este preço ${ }^{5}$ será estimado com base na Pesquisa de Emprego e Desemprego da Região Metropolitana de Salvador (PED-RMS) para o período de outubro de 1996 a dezembro de 2002.

$\mathrm{R}_{\mathrm{j}}$ é o valor presente do fluxo de renda vitalícia total dos candidatos ao curso $\mathrm{j}$, descontada à taxa instantânea de juro $\beta$, a partir do primeiro ano de formatura; refere-se à renda total dos candidatos ao curso $\mathrm{j}$, calculada a partir de dados da PED; ou seja, $R_{j}=\sum_{\mathrm{i}=1, \ldots, m} R_{\mathrm{ij}}$.

Formando-se o Lagrangiano a partir de uma função de utilidade Cobb-Douglas:

${ }^{5}$ Ao longo de todo este artigo, custo de oportunidade e preço são termos intercambiáveis. 


$$
L(c ; \lambda) \equiv \prod_{j=1, \ldots, n+p} c_{j}^{a j}+\lambda\left[R_{j}^{1}-\Sigma_{j=1, \ldots, n+p} p_{j} \cdot c_{j}\right]
$$

onde $\mathbf{c}=\left(\mathbf{c}_{1}, \mathbf{c}_{2}, \ldots, \mathbf{c}_{\mathrm{n}+\mathrm{p}}\right)$ é o vetor de bens, $\Pi_{\mathrm{j}=1, \ldots, \mathrm{n}+\mathrm{p}}$ é o produtório com $\mathrm{j}$ variando de 1 a $n+p$, onde $n$ é a quantidade total de cursos que compõem o conjunto de escolhas possíveis e $\mathrm{p}-\mathrm{n}+1$ é a quantidade demandada por outros bens.

Para que a função de demanda seja homogênea de grau zero, a condição $\sum_{j=1, \ldots, n+p} a_{j}=1,(a j>0)$ deve ser satisfeita. A função de demanda ser de grau zero é importante porque significa que se os preços variam de $\theta$, a renda também variará de $\theta$; em outras palavras, a escolha do consumidor não será afetada por esta razão. Ademais, uma vez que se trata de renda vitalícia, é bastante plausível que o indivíduo esgote toda a sua renda, ou seja, é satisfeita a lei de Walras, o que significa que $R_{1}{ }_{j}=\Sigma_{j=1, \ldots, n+p}$ $p_{j} \cdot c_{j}$. Lembre-se também que o indivíduo consome outros bens, $c_{n+1}, \ldots, c_{n+p}$.

É claro que durante determinados momentos o indivíduo pode ser um poupador ou "despoupador" líquido, financiando ou remunerando os seus déficits ou superávits, respectivamente, à taxa de juro $\beta$. No caso de financiamento da educação por meio do mercado de capitais, $\beta$ seria a taxa de juro máxima que o indivíduo deveria arcar de modo a amortizar a dívida. Dada a imperfeição do mercado de capitais e grandes externalidades positivas da educação, torna-se especialmente importante para o País a existência do FIES como mecanismo de financiamento da educação.

Pelas condições de primeira ordem:

$$
\begin{aligned}
& \left(\partial L / \partial c_{j}\right)=\left(a_{j} / c_{j}\right) \prod_{j=1, \ldots, n+p} c_{j}{ }^{\alpha j}-\lambda p_{j}=0 \\
& (\partial L / \partial \lambda)=R_{j}-\Sigma_{j=1, \ldots, n+p} p_{j} \cdot c_{j}=0
\end{aligned}
$$

Resolvendo-se o sistema formado por (4) e (5), extraem-se as funções de demanda pelo curso j:

$$
\begin{aligned}
& c_{j}^{d}=\left(a_{j} R_{i j}^{1 j}\right) /\left[p_{j} .\left(\sum_{j=1, \ldots, n+p} a_{j}\right)\right]=a_{j} R_{j}^{1} / p_{j} \\
& \lambda=\left[\operatorname { e x p } \left(\sum_{j=1, \ldots, n} \ln \left(a_{j} R_{j} / p_{j}\left(\sum_{j=1, \ldots, n+p} a_{j}\right) \cdot a_{j}\right] / R_{j}\right.\right.
\end{aligned}
$$

onde $\lambda$ é a utilidade marginal da renda vitalícia. 
A condição de segunda ordem para um máximo é satisfeita na medida em que a função de utilidade Cobb-Douglas é quase côncava.

\subsection{Modelos para cálculo do custo de oportunidade e da renda vitalícia do candidato}

O Diagrama 1 do fluxo de caixa estiliza a decisão que o candidato ao concurso vestibular se depara. Ele é apenas ilustrativo e serve para fixar as idéias quanto ao cálculo de $\mathrm{p}_{\mathrm{ij}}$ e $\mathrm{R}_{\mathrm{ij}}$. Conforme este diagrama, o indivíduo encontra-se diante de um dilema: se ele resolve fazer a faculdade, ele sacrifica a renda $R_{11}$ pelo período de $D$ anos em troca de uma renda futura maior, com certa probabilidade. A probabilidade de o recém- formado não encontrar trabalho é menor do que aquele indivíduo que apenas tem o curso médio completo, mas é existente. Neste trabalho não se incorporam as probabilidades associadas às incertezas de uma decisão. Ademais, em verdade, as rendas $R_{\mathrm{ij}}$ e $\mathrm{p}_{\mathrm{ij}}$ não são constantes no tempo, mas côncavas.

\section{Diagrama 1: Fluxo de Caixa da decisão do indivíduo que deseja ingressar na Universidade ${ }^{6}$ :}

$$
\mathrm{R}_{\mathrm{D}+\mathrm{E}}
$$

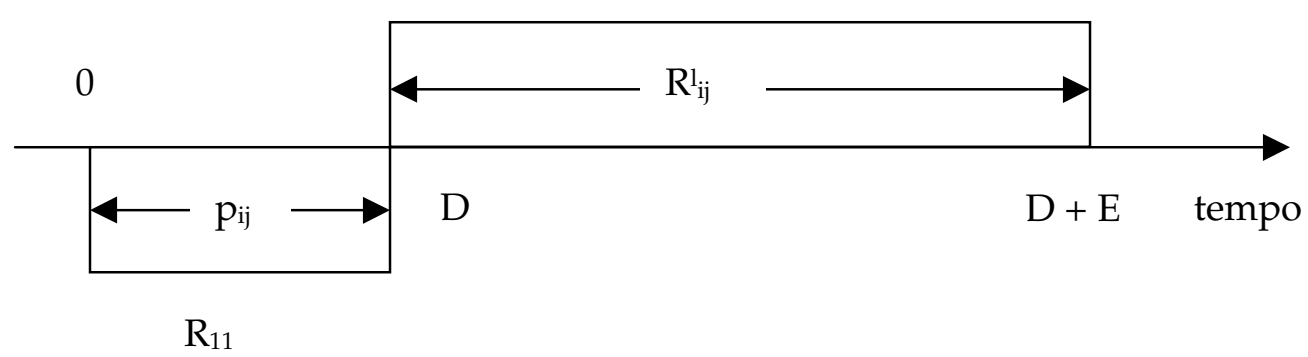

Onde,

Zero é o instante em que o indivíduo decide-se por ingressar na Universidade;

6 No caso do indivíduo que trabalha, considerou-se, para simplificar, que o seu custo de oportunidade fosse zero, tornando nula a área $\mathrm{p}_{\mathrm{ij}}$. 
$\mathrm{R}_{\mathrm{D}+\mathrm{E}}$ é a renda média obtida após a conclusão do curso superior de duração de $\mathrm{D}$ anos; neste caso, além do acúmulo de capital humano proveniente de investimentos em ensino superior, o indivíduo ingressa no mercado de trabalho e começa a acumular capital humano em decorrência de sua experiência no mercado de trabalho, E;

$\mathrm{R}_{11}$ é a renda média que o indivíduo i obteria caso ele optasse por trabalhar ao invés de ingressar na Universidade e possuísse uma capacitação correspondente ao ensino médio completo (onze anos de escolaridade);

O cálculo do custo de oportunidade do indivíduo i no curso j, $\mathrm{p}_{\mathrm{ij}}$, seria dado por:

$$
p i j=\int_{0}^{D_{i j}} R(t) \cdot \exp (-\beta \cdot t) \cdot d t
$$

Onde

$\beta$ é a taxa instantânea de juros utilizada para descontar o fluxo de renda do indivíduo, $\mathrm{R}(\mathrm{t})$;

$D_{i j}$ é a duração efetiva do curso j escolhido pelo aluno i;

$\exp$ ( ) é a função exponencial, e

$$
R(t)=\left\{\begin{array}{l}
\text { Ro.exp[y.(t-S-7)+ } \left.\delta \cdot(t-S-7)^{2}+\psi \cdot s e x o+\mu \cdot b r a n c a+\text { v.parda }+\Sigma_{\mathrm{s}} \varphi(\mathrm{S}) \cdot \mathrm{D}(\mathrm{S})\right] \\
\mathrm{S}=1,2, \ldots, 11 \mathrm{e} 0 \leq \mathrm{t} \leq \mathrm{D}_{\mathrm{ij}} ; \\
0, \text { caso contrário. }
\end{array}\right.
$$

Onde,

Ro é a renda anual do cidadão com zero ano de escolaridade; 


$$
\mathrm{E}=\mathrm{t}-\mathrm{S}-7^{7}
$$

e,

E é a experiência no mercado de trabalho do candidato; $\mathrm{S}$ é a escolaridade do indivíduo em anos e té a idade do indivíduo.

Sexo, branca e parda são variáveis binárias que assumem o valor 1 quando o candidato for do sexo masculino, da raça auto-declarada branca ou parda, e assumem o valor zero no caso contrário. A variável omitida é a referente à etnia negra.

$\mathrm{D}(\mathrm{S})$ é uma variável binária que assume o valor um quando o indivíduo tem $\mathrm{S}$ anos de escolaridade, e o valor zero, caso contrário.

Substituindo-se (9) em (8), resolvendo-se a integral e simplificando, chega-se à equação (11):

$$
\begin{aligned}
& \mathrm{p}_{\mathrm{ij}}=\operatorname{Ro} \cdot \mathrm{D}_{\mathrm{ij}} \cdot \exp \left[\left(4 \cdot \psi \cdot \delta \cdot \operatorname{sexo}+4 \cdot \text {. } \cdot \delta \cdot \operatorname{parda}+4 \cdot \delta \cdot\left(\Sigma_{\mathrm{s}=1 . .17} \varphi(\mathrm{S}) \cdot \mathrm{D}(\mathrm{S})\right)+\ldots\right.\right. \\
& \left.\left.\ldots+4 . \mu \delta \text {.branca }-\beta^{2}-4 . \beta . \delta . S-28 . \beta . \gamma+2 . \beta \gamma-\gamma^{2}\right) /(4 . \delta)\right]
\end{aligned}
$$

Os parâmetros $\gamma, \delta, \Psi, v, \mu$ e $\varphi(S)(S=1,2, \ldots, 17)$ da equação (11) serão estimados a partir da equação (12) de salários ampliada à la MINCER (1974):

$\operatorname{Ln} R(t)=\ln R o+\gamma E+\delta E^{2}+\psi \cdot \operatorname{sexo}+\mu \cdot b r a n c a+$ v.parda $+\Sigma_{s=1.17} \varphi(S) \cdot D(S)+\varepsilon^{8}$

Por sua vez, o cálculo da renda vitalícia, $\mathrm{R}_{\mathrm{ij}}$, é obtido a partir da função integral (13):

7 Conforme demonstrado em Avena (2000), esta especificação apresenta resultados mais condizentes com o bom senso do que simplesmente se utilizar a idade como proxy para experiência no mercado de trabalho.

$8 \varepsilon$ é um termo estocástico suposto normalmente distribuído, com média $\mu$ e homoscedástico. 


$$
R_{1 j}=\int_{0}^{D_{i j}+E} R^{1}(t) \cdot \exp (-\beta \cdot t) \cdot d t
$$

Onde,

$\mathrm{R}^{1}(\mathrm{t})$ é a função da renda vitalícia (fluxo anual) do indivíduo após ter cursado $\mathrm{D}_{\mathrm{ij}}$ anos de faculdade;

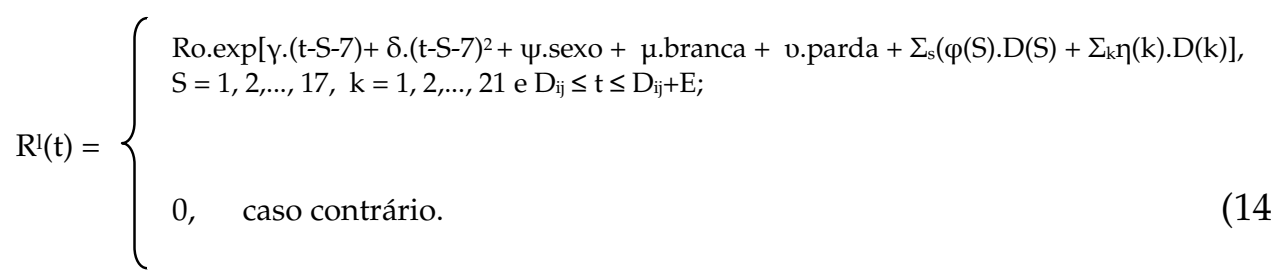

Onde $\eta(k)(k=1,2, \ldots, 21)$ são os coeficientes a serem estimados relativos a cada um dos cursos (profissões) para os quais há dados de renda disponível e $\mathrm{D}(\mathrm{k})$ são variáveis binárias que assumem o valor um se o candidato é formado no curso k, e assume o valor zero, em caso contrário.

Substituindo-se (14) em (13), resolvendo e simplificando, resulta na equação 15:

$$
\begin{aligned}
\mathrm{R}_{\mathrm{ij}}=\operatorname{Ro} .\left(\mathrm{D}_{\mathrm{ij}}+\mathrm{E}\right) \cdot \exp \left\{\left[\left(4 \cdot \delta \cdot \Sigma_{\mathrm{s}}(\varphi(\mathrm{S}) \cdot \mathrm{D}(\mathrm{S})+4 \cdot \delta \cdot \psi \cdot \operatorname{sexo}+4 \cdot v \cdot \delta \cdot \operatorname{parda}+\ldots\right.\right.\right. \\
\left.\left.\quad \ldots+4 \cdot \delta \cdot\left(\Sigma_{\mathrm{k}} \eta(\mathrm{k}) \cdot \mathrm{D}(\mathrm{k})\right)+4 . \mu \cdot \text { branca }-\gamma^{2}+2 \cdot \beta \gamma-\beta^{2}-4 \cdot \beta \cdot \delta \cdot S\right] /(4 . \delta)\right\}
\end{aligned}
$$

$9 \mathrm{p}_{\mathrm{ij}}$ e $\mathrm{R}_{\mathrm{ij}}$ podem ser interpretados como variáveis de estoque; no caso de $\mathrm{R}_{\mathrm{ij}}$ seria o estoque de capital humano do indivíduo i e profissão j. 
Os parâmetros $\gamma, \delta, \Psi, v, \mu, \varphi(S)(S=13,14, \ldots, 17)$ e $\eta(k)(k=1,2, \ldots, 21)$ da equação (15) serão estimados a partir da equação de salários ampliada (16):

$$
\begin{aligned}
\operatorname{Ln} R(t)=\ln R o+\gamma E+\delta E^{2}+\psi \cdot \operatorname{sexo}+ & \mu \cdot \text { branca }+ \text { v.parda }+\ldots \\
& \ldots+\Sigma_{\mathrm{s}=1.17} \varphi(\mathrm{S}) \cdot \mathrm{D}(\mathrm{S})+\Sigma_{\mathrm{k}} \eta(\mathrm{k}) \cdot \mathrm{D}(\mathrm{k})+\varepsilon^{10}
\end{aligned}
$$

\section{OS DADOS}

Foram utilizadas duas bases de dados para a realização deste trabalho. A base da Pesquisa de Emprego e Desemprego da Região Metropolitana de Salvador (PED-RMS) e a base de dados do Vestibular da UFBa, cada uma com um número total de 475.188 e 21.459 casos, respectivamente.

A base da PED-RMS utilizada vai do período de outubro de 1996 a dezembro de 2002; quanto à base do Vestibular, engloba os casos a partir de 1993 até 2001, exceto os anos de 1996, 1999 e 2000, por não se encontrarem disponíveis. Nas análises realizadas, nem todos os casos são utilizados, por haver dados indisponíveis para certas variáveis, sendo estes casos excluídos da análise.

Os valores monetários relativos às rendas e preços encontram-se em base constante de dezembro de 2002, segundo o índice de preços, IPC-SEI, eliminando-se, assim, o efeito da inflação acumulada de pouco mais de 50\% no período, a partir de outubro de 1996 até dezembro de 2002. Assim, toda a análise realizada neste trabalho é em termos reais, isto é, pelo poder aquisitivo da moeda.

A despeito de a base de dados sobre o Vestibular da UFBa ter muitas informações sobre cada um dos candidatos inscritos, optou-se, neste artigo, por trabalhar com a base dos candidatos aprovados por dispor de informações essenciais para as estimações realizadas. Não se utilizou a base dos inscritos pela necessidade que haveria de serem imputados muitos dados faltantes, deixando-se para fazer isto em trabalho posterior.

A estimação das rendas vitalícias ${ }^{11}$ foi possível pelo fato de a base da PED-RMS dispor de informações por profissão, o que permitiu calcular

\footnotetext{
$10 \varepsilon$ é um termo estocástico suposto normalmente distribuído, com média $\mu$ e homoscedástico.

11 Para efeito de cômputo da renda vitalícia, considerou-se um período de experiência de 30 e de 35 anos para a mulher e para o homem, respectivamente.
} 
a renda das seguintes áreas profissionais: Arquitetura, Engenharias Civil, de Minas, Elétrica, Mecânica, Química, e Sanitária e Ambiental (todas estas estimadas em conjunto); Administração, Agronomia, Direito, Análise de Sistemas (como proxy para Ciência da Computação), Biblioteconomia e Museologia (rendas e preços estimados em conjunto), Arqueologia, Antropologia, Geografia e Sociologia (rendas e preços estimados em conjunto), Biologia, Odontologia, Economia, Enfermagem, Matemática e Estatística, Geologia, Jornalismo, Medicina, Nutrição, Música, Educação Física, Psicologia, Medicina Veterinária e Química.

As profissões a que se fez menção a estimação conjunta se justificou porque não havia como separá-las na base da PED-RMS uma vez que a codificação dessas profissões é a mesma; por igual razão fez-se o mesmo com Arquitetura e Engenharias, Matemática e Estatística (estas duas últimas, por haver apenas um único profissional Matemático pesquisado, resolveu-se combiná-las). Assim, tem-se um total de 21 cursos cujas demandas serão estimadas.

\section{RESULTADOS DOS MODELOS ECONOMÉTRICOS}

Nesta seção, algumas funções de demanda serão estimadas. Inicialmente, porém, se apresenta a equação que estima os coeficientes da equação (9) para se calcular os custos de oportunidade dos alunos e, em seguida, apresenta-se a estimação da equação (12), o que permite calcular a renda vitalícia do aluno.

O modelo da equação (11) (referente ao custo de oportunidade) estimado é o seguinte:

$$
\begin{gathered}
\operatorname{Ln}\left(p_{i j}\right)=6,267+0,06404 \cdot E-0,000887 \cdot E^{2}+0,581 \cdot \text { Sexo }+0,313 \cdot \text { branca }+0,106 \cdot \text { parda }+\ldots \\
\ldots+0,139 \cdot \mathrm{D}(1)+\ldots+1,711 \cdot \mathrm{D}(13)+1,823 \cdot \mathrm{D}(14)+2,272 \cdot \mathrm{D}(15)+\ldots \\
\ldots+2,645 \cdot \mathrm{D}(16)+2,785 \cdot \mathrm{D}(17)
\end{gathered}
$$

Os coeficientes estimados são todos significativamente diferentes de zero, ao nível de confiança de $0 \%$, praticamente; ademais, a estatística $\mathrm{F}$ encontrada foi de 7.063,4, também ao nível de significância igual a zero. $\mathrm{O}$ coeficiente de determinação múltipla, $\mathrm{R}^{2}$, foi bastante elevado e igual a $51,7 \%$. O número de observações utilizados nesta estimativa foi de 145.429.

Os coeficientes das variáveis $\mathrm{D}(2), \mathrm{D}(3), \ldots, \mathrm{D}(12)$ foram suprimidos porque somente interessam-se pelos $\mathrm{D}(13), \mathrm{D}(14), \ldots, \mathrm{D}(17)$ para cálculo do 
custo de oportunidade do aluno, pois é o período em que ele se encontra na Universidade. $\mathrm{D}(12)$ é suprimido, pois nenhum aluno se formou com apenas um ano de faculdade.

O modelo referente à renda vitalícia estimado pela equação (15) é o da equação (18):

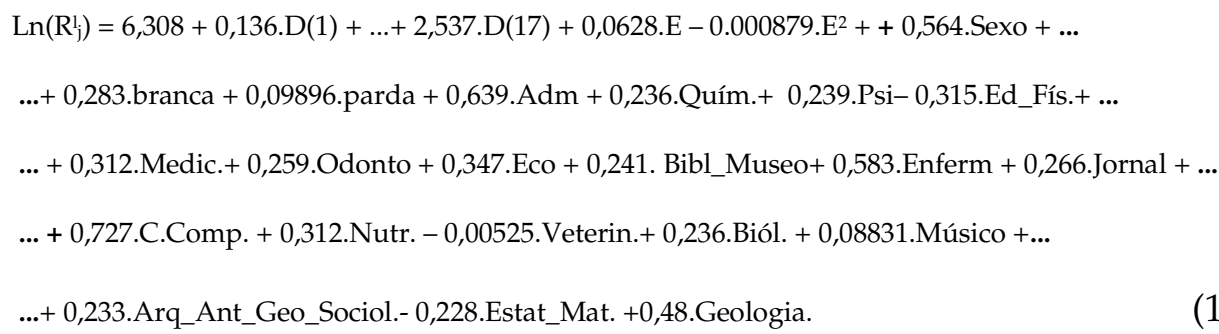

Os coeficientes estimados são todos significativamente diferentes de zero, a exceção dos relativos às variáveis Agronomia, Medicina Veterinária e Arqueologia, Antropologia, Geografia e Sociologia (estas quatro últimas representadas pela variável Arq_Ant_Geo_Sociol.) ao nível de confiança de $0 \%$, praticamente; ademais, a estatística $\mathrm{F}$ encontrada foi de 3.821,40, também ao nível de significância igual a zero. $O$ coeficiente de determinação múltipla, $\mathrm{R}^{2}$, foi bastante elevado e igual a 53,1\%. O número de observações utilizados nesta estimativa foi de 145.429.

\subsection{Modelos de Demanda}

O primeiro modelo de demanda é o da equação (20), obtida da equação de demanda (6) após se tomar o logaritmo neperiano:

$$
\operatorname{Ln} c_{j}{ }^{d}=\ln \alpha+\ln R_{j}{ }^{1}-\ln p+\varepsilon
$$

Onde,

$c_{\mathrm{j}}^{\mathrm{d}}$ é a quantidade demandada agregada pelo curso j por ano;

$\mathrm{R}_{\mathrm{j}} \mathrm{l}$ é a renda vitalícia após fazer-se $\mathrm{R}_{\mathrm{j}}{ }^{\mathrm{l}}=\sum_{\mathrm{j}=1, \ldots, \mathrm{n}} \mathrm{R}_{\mathrm{ij}}$;

$\varepsilon$ é o termo estocástico com as hipóteses usuais. 
O modelo da equação (19) estimado é o da equação (20) com as estatísticas correspondentes (a amostra em referência é composta apenas pelos indivíduos que não trabalham):

$$
\begin{gathered}
\operatorname{Ln} c_{j}{ }^{d}=-5,621-0,251 . \ln p_{j}+0,797 \cdot \ln R_{j}^{1_{j}} \\
(-56,07)(-22,4)
\end{gathered}
$$

Os sinais dos coeficientes apresentaram-se conforme o esperado, ou seja, há correlações negativa e positiva, respectivamente, com respeito ao preço e à renda. Isto demonstra que educação superior não é um bem de Giffen. Além disso, o coeficiente de determinação múltipla, $\mathrm{R}^{2}$, mostrou-se excepcionalmente elevado, igualando-se a 91,1\%; isto demonstra que apenas $8,9 \%$ da variabilidade da quantidade demandada não foram explicadas por apenas duas variáveis, preço e renda. As estatísticas t, abaixo dos coeficientes estimados, são muito elevadas, demonstrando a significância estatística das duas variáveis e do intercepto, mesmo ao nível de significância de 0\%. Essa estimativa envolveu 3.208 observações. Ressalte-se que essas estimativas envolveram apenas os indivíduos não empregados, para quem, portanto, o ato de estudar representa um custo de oportunidade máximo; por conseguinte, o coeficiente da variável relativa a preço é relativamente mais elevado.

O fato de o coeficiente de determinação múltipla ser tão elevado, $91,3 \%$, merece um comentário à parte. Isto mostra a aderência do modelo à realidade dos dados com bastante força, o que denota que os indivíduos que procuram fazer um curso superior estão muito preocupados com a trajetória de renda que a sua profissão escolhida poderá proporcionar (haja vista a significância estatística da variável renda), mas sem se descuidar do sacrifício que terá que fazer por se ausentar do mercado de trabalho para estudar. Assim, espera-se que os dados confirmem o bom senso, de que quanto mais longos os cursos, menor a demanda por eles.

A função de demanda por ensino superior (20) permite calcular a elasticidade-preço da demanda, $\varepsilon_{\mathrm{pp}}$, a elasticidade-juro da demanda, $\varepsilon_{\mathrm{p} \beta}$, a elasticidade-escolaridade da demanda, $\varepsilon_{\mathrm{pS}}$, e a elasticidade-renda da demanda, $\eta$. 


$$
\begin{aligned}
& \varepsilon_{p p}=\left(\partial \ln c_{j} / \partial \ln p_{j}\right)=-0,251 \\
& \eta=\left(\partial \ln c_{j}^{d_{j}} / \partial \ln R_{j}^{l}\right)=0,797 \\
& \varepsilon_{p \beta}=\left[\left(\partial c_{j}^{d} / \partial p_{j}\right) \cdot\left(\partial p_{j} / \partial \beta\right)+\left(\partial c_{j}^{d} / \partial R_{j}^{l}\right)\left(\partial R_{j}^{1} / \partial \beta\right)\right] \cdot\left(\beta / c_{j}^{d}\right)=-17,85 \\
& \varepsilon_{p S}=\left[\left(\partial c_{j}^{d} / \partial p_{j}\right) \cdot\left(\partial p_{j} / \partial S\right)+\left(\partial c_{j} / \partial R_{j}^{l}\right)\left(\partial R_{j}^{l} / \partial S\right)\right] \cdot(S / c)=-0,56
\end{aligned}
$$

A equação (21) nos informa que para cada aumento de $1 \%$ no custo de oportunidade do aluno, a quantidade demandada reduz-se $0,251 \%$. Trata-se de um bem, portanto, cuja elasticidade é inelástica. Já a equação (22) nos informa que o ensino superior público é um bem normal, pois a sua elasticidade-renda é positiva; além disso, para cada $1 \%$ de acréscimo na renda-vitalícia, a demanda por ensino superior aumenta 0,797\%.

A elasticidade-juro da demanda (equação 23) é extremamente alta, mostrando a alta sensibilidade da demanda ao aumento da taxa real de juro. Calculada esta elasticidade no ponto $\beta=11,3329 \%$, a demanda cai de quase $18 \%$ para cada $1 \%$ de aumento de $\beta$. Este efeito é decorrência da redução extremamente alta do valor presente da renda vitalícia. Se o aluno tiver essa percepção no seu cálculo econômico, a demanda se reduz neste valor.

A equação 24, calculada para a escolaridade de 11 anos, quando o indivíduo decide fazer, ou não, o Vestibular, revela que a cada ponto percentual de anos de estudo, o aluno reduz a sua demanda em 0,56\%. Uma vez que a escolaridade não é medida em percentagem, mas em anos, se o aluno passa de 11 para 12 anos de escolaridade, quando há um aumento de $9,1 \%$ na escolaridade, a demanda para este aluno será menor em 5,1\%. Mais interessante do que isto, entretanto, é que a elasticidadeescolaridade da demanda é crescente, em valor absoluto, com a escolaridade; ou seja, quanto maior a escolaridade do aluno, mais sensível se torna a demanda, chegando a $-0,72$ quando o aluno alcança 14 anos de escolaridade. Isto parece revelar que se torna cada vez mais difícil recuperar um aluno que não completou os seus estudos superiores.

Com base na equação 20, calculou-se o tempo ótimo de duração de um curso. Esse tempo ótimo corresponde a 63,8\% do tempo de experiência no mercado de trabalho. Por exemplo, um aluno que tem 25 anos, pela equação (10), ele teria 7 anos de experiência e a duração ótima de seu curso terá sido de 4,5 anos. Esta relação somente faz sentido para valores de idade $t$, tais que $18 \leq t \leq 30$.

Além disso, essas informações permitem à Universidade planejar-se quanto à oferta de vagas. Por exemplo, supondo um crescimento anual de 
5\% da renda nos próximos 5 anos, haveria um aumento de demanda superior a $14 \%$ ao final desse período.

A função de demanda permite inferir o porquê de haver maior demanda por ensino superior quando aumenta o desemprego no mercado de trabalho (ou seja, o ensino superior é contra-cíclico); com efeito, uma maior taxa de desemprego reduz o custo de oportunidade do aluno, seja diretamente, via desemprego, anulando o custo de oportunidade, seja indiretamente, reduzindo os salários monetários pela maior pressão de oferta de mão de obra mais qualificada; já a renda vitalícia, sendo uma renda de longo prazo, não seria afetada por movimentos cíclicos de curto prazo da economia. A taxa de desemprego por profissão não é um dado disponível na base da PED-RMS, necessitando ser calculada.

Um outro modelo estimado é o da equação (25) (em amostra com indivíduos que trabalham ou não trabalham):

$$
\begin{aligned}
& \ln \mathrm{c}_{\mathrm{j}}^{\mathrm{d}}=-6,541+1,594 \mathrm{D}+0,723 . \ln \mathrm{R}_{\mathrm{j}}-0,182 \ln \mathrm{p}_{\mathrm{j}} \text { ， } \\
& (-125,4) \quad(15,08) \quad(229,3) \quad(-15,0)
\end{aligned}
$$

onde D é uma variável binária que assume o valor zero se o indivíduo trabalha e o valor um, caso ele não trabalhe durante o curso. Como se pode observar, as estatísticas $t$ (abaixo dos coeficientes estimados) apresentam-se muito elevadas, atestando a hipótese de que sejam significativamente diferentes de zero, a um nível de significância de $0 \%$. Ademais, a estatística F calculada foi de 20.553,6 (significância igual a $0 \%$ ). O R $\mathrm{R}^{2}$ também foi extremamente elevado e igual a 90,4\%.

A elasticidade-renda na especificação da equação (25) não se altera significativamente, mas a elasticidade-preço se reduz, em valor absoluto, mostrando-se ainda mais inelástica. Esta redução acentuada no coeficiente dos preços decorre da alta correlação de Pearson entre esta variável e a variável binária, trabalha ou não trabalha $(\mathrm{r}=99,4 \%)$. Assim, resolveu-se fazer mais uma estimativa apenas com a amostra dos que trabalham. Os percentuais dos que não trabalham ou trabalham durante o curso são de $69,7 \%$ e $30,3 \%$, respectivamente.

Para a amostra de candidatos que trabalham, o resultado é o da equação (26):

$$
\begin{aligned}
\ln c_{j}{ }^{d}= & -5,815+0,679 \ln R_{j}{ }^{1} \\
& (-83,8) \quad(161,5)
\end{aligned}
$$


Observe-se que na equação (26) não aparece a variável preço, pois, como mencionado anteriormente, foi atribuído o valor zero ao custo de oportunidade de quem trabalha. As estatísticas $t$ mostraram-se muito elevadas, atestando a diferença de zero tanto do intercepto quanto do coeficiente angular, ao nível de significância de $0 \%$; a estatística $\mathrm{F}$ foi igual a $26.081,3$ a $0 \%$ de significância e o $\mathrm{R}^{2}$ foi extremamente elevado, de $88,7 \%$, atestando o poder de explicação da variável renda vitalícia.

Testaram-se as variáveis binárias se o pai ou a mãe do candidato tinham curso superior incompleto ou completo (quando assumia o valor igual a 1) contra o caso contrário, quando tinham escolaridade menor ou igual ao ensino médio; estas variáveis, entretanto, não se mostraram estatisticamente significativas. Quando excluída apenas a variável relativa à escolaridade da mãe, o fato de o pai ter o nível superior completo ou incompleto mostrou-se estatisticamente significativa. Curiosamente, os coeficientes estimados são negativos, denotando que quanto maior a escolaridade do pai e da mãe, menor a demanda por ensino superior.

Realizaram-se estimativas por área, nas áreas de estudo 1, 2 e 3. As áreas 4 e 5 não puderam ser estimadas por não existirem dados sobre renda na base da PED-RMS. A área 1 engloba os cursos que têm uma fundamentação matemática mais forte, como Arquitetura, Engenharias, Ciência da Computação, Geofísica, Matemática, Estatística, etc. A área 2 engloba os cursos de ciências médicas e biológicas, Farmácia, Enfermagem, Nutrição, Odontologia, etc. A área 3, por sua vez, corresponde aos cursos de Administração, Ciências Econômicas e Contábeis, Educação Física, Direito, Jornalismo, Psicologia, etc. Os resultados encontrados foram os seguintes:

Para os cursos da área 1:

$$
\begin{aligned}
& \ln c_{\mathrm{j}}^{\mathrm{d}}=-4,418+0,583 \ln \mathrm{R}_{\mathrm{j}}{ }^{\mathrm{l}}-0,0025 \ln \mathrm{p}_{\mathrm{j}} \\
& (-61,3) \quad(131,05) \quad(-1,96)
\end{aligned}
$$

O coeficiente da variável preço mostrou-se bastante reduzido, mostrando a quase total inelasticidade dos cursos dessa área aos custos de oportunidade, porém, a estatística $\mathrm{t}$ também foi reduzida ao nível de significância de $5 \%$. Provavelmente este resultado se deve à grande agregação dos valores nesta área, pois na base da PED-RMS não se faz distinção entre Arquitetura e Engenharias, conforme mencionado anteriormente; a elasticidade-renda foi de 0,583, mostrando-se mais reduzida do que na especificação em todas as áreas. 
Os resultados da área 2 foram:

$$
\begin{aligned}
& \ln c_{j}{ }^{d}=-7,244+0,772 \ln R_{j}{ }^{l}+0,0036 \ln p_{j} \\
& (-124,1)(226,25) \quad(5,02)
\end{aligned}
$$

As estatísticas $\mathrm{t}$ e $\mathrm{F}$ foram todas significativas ao nível de $0 \%$. Um resultado esdrúxulo, no entanto, é o coeficiente do preço ser positivo, denotando que quanto maior o custo de oportunidade do candidato à área 2, maior a quantidade demandada. Aliado a isto, o coeficiente da renda é positivo. Os cursos da área 2 para serem considerados um bem de Giffen teriam que ser bens inferiores, o que não é o caso.

Quanto à área 3, os resultados encontrados são mostrados na equação 29:

$$
\begin{aligned}
\ln c_{j}{ }^{d}= & -5,802+0,688 \ln R_{j}{ }^{1}-0,0048 \ln p_{j} \\
& (-76,2) \quad(152,76) \quad(-3,15)
\end{aligned}
$$

O R 2 mostrou-se bastante elevado e igual a $92,8 \%$ e as estatísticas t e $\mathrm{F}$ atestam a diferença de zero dos coeficientes estimados ao nível de significância de $0 \%$. Os coeficientes da renda e do preço também apresentam os sinais esperados, sendo positivo e negativo, respectivamente. Mas a elasticidade-preço da demanda mostra-se muito reduzida, atestando a baixa sensibilidade da demanda a variações de preço.

Uma vez que cada área representa um risco associado à escolha, resolveu-se estimar a demanda onde cada área é uma variável binária; deste modo, as variáveis relativas às áreas 1 e 2 (representadas na equação por A1 e A2, respectivamente) assumem valor um se o indivíduo escolheu uma carreira nessas áreas e assume o valor zero, caso contrário. A área 3 é a variável omitida. O resultado é o da equação 30:

$$
\begin{aligned}
& \ln c_{j}{ }^{d}=-5,647+0,677 \ln R_{j}{ }^{1}-0,0021 \ln p_{j}-0,32 A 1+0,0795 A 2 \\
& (-122,6) \quad(248,12) \quad(-2,62) \quad(-39,7) \quad(9,40)
\end{aligned}
$$


A equação (30) revela o que era esperado: há uma menor demanda pela área 1, por representar um maior risco de não se concluir o curso, pela exigência de raciocínio mais abstrato, e uma maior demanda por cursos da área 2. As estatísticas estimadas foram muito significativas, ao nível de $0 \%$, a exceção da variável preço que foi significativa ao nível de $9 \%$; ademais, o $\mathrm{R}^{2}$ foi de $93,2 \%$ com uma amostra de 6.534 observações.

Duas outras especificações foram testadas com amostras parciais de quem trabalha e entre aqueles que não trabalham, adicionando-se a variável ln (renda familiar). Os resultados mostram que a renda familiar não é significativa para quem não trabalha (quem não precisa trabalhar é porque tem uma renda familiar que assim o permite) (equação 32), mas é significativa para quem trabalha (equação 33); além disso, quanto maior a renda de quem trabalha, menor a demanda por ensino superior, pois o custo de oportunidade é crescente com a renda. Com efeito:

$$
\begin{aligned}
& \operatorname{lnc} \mathrm{j}^{\mathrm{d}}=-5,606+0,778 \ln \mathrm{R}^{1}-0,252 \ln \mathrm{p}+0,000022 \text { Escore }+0,00279 \ln R e n d a \\
& (-54,26) \quad(-22,33) \quad(2,62) \quad(0,48)
\end{aligned}
$$

A equação 31 revela uma coisa interessante: primeiro, uma vez que se refere a uma amostra de candidatos que não trabalham, esses indivíduos buscam profissões em que o escore mínimo para ser aprovado é menos significativo do que os que trabalham (vide equação 33); segundo, a renda familiar não é estatisticamente significativa ao nível de $63 \%$. Ou seja, quem não trabalha não o faz porque não precisa.

$$
\begin{aligned}
\ln c_{j}{ }^{d}= & -5,765+0,668 \ln R^{1}+0,0000425 \text { Escore }-0,0138 \ln R e n d a \\
& (-72,51) \quad(139,56) \quad(5,64)
\end{aligned}
$$

Em uma especificação bem mais abrangente, incluindo-se outras variáveis, obtêm-se as parcelas de uma estimativa feita levando-se em conta se o candidato pertence (assume valor 1) ou não pertence (assume valor zero) ao conjunto de determinada profissão. Os resultados são mostrados na Tabela 1 e os comentários aqui se reportam a ela. 


\section{Tabela 1: Resultados da estimação da equação de demanda completa}

Variável dependente: Ln quantidade demanda por ano

\begin{tabular}{|c|c|c|c|c|}
\hline & $\begin{array}{l}\text { Coeficientes } \\
\text { estimados }\end{array}$ & Erro-padrão & Estatística t & Significância \\
\hline Intercepto & -6.1810300 & 0.057 & -108.759 & 0.000 \\
\hline Ln custo de oportunidade & 0.0002642 & 0.001 & 0.470 & 0.638 \\
\hline Ln estoque de capital humano & 0.7016559 & 0.003 & 204.122 & 0.000 \\
\hline Ln renda familiar & 0.0019395 & 0.002 & 0.846 & 0.398 \\
\hline Administração & -0.2965606 & 0.013 & -22.744 & 0.000 \\
\hline Agronomia & -0.1809143 & 0.010 & -17.521 & 0.000 \\
\hline Arquitetura e Engenharias & -0.5235126 & 0.008 & -66.881 & 0.000 \\
\hline Ciência da Computação & -0.4235756 & 0.014 & -30.640 & 0.000 \\
\hline Direito & 0.1613450 & 0.014 & 11.237 & 0.000 \\
\hline Economia & -0.2110621 & 0.011 & -19.153 & 0.000 \\
\hline Educação Física & 0.0881588 & 0.013 & 6.958 & 0.000 \\
\hline Enfermagem & 0.0585993 & 0.012 & 4.797 & 0.000 \\
\hline Geologia & -0.5530548 & 0.012 & -44.528 & 0.000 \\
\hline Jornalismo & -0.6622110 & 0.016 & -42.255 & 0.000 \\
\hline Matemática / Estatística & 0.0484575 & 0.008 & 5.810 & 0.000 \\
\hline Medicina & 0.0762014 & 0.015 & 5.174 & 0.000 \\
\hline Medicina Veterinária & 0.1772547 & 0.011 & 15.967 & 0.000 \\
\hline Música & -0.7002232 & 0.018 & -39.843 & 0.000 \\
\hline Nutrição & 0.1490365 & 0.012 & 12.413 & 0.000 \\
\hline Odontologia & 0.2132464 & 0.013 & 16.195 & 0.000 \\
\hline Psicologia & 0.2463574 & 0.013 & 19.281 & 0.000 \\
\hline Escore global após classificação & 0.0000193 & 0.000 & 6.377 & 0.000 \\
\hline Capital (binária) & 0.0048762 & 0.007 & 0.737 & 0.461 \\
\hline Masculino (binária) & 0.0025601 & 0.004 & 0.647 & 0.518 \\
\hline Solteiro (binária) & 0.0034183 & 0.007 & 0.487 & 0.627 \\
\hline $\begin{array}{l}\text { Superior completo ou incompleto } \\
\text { da mãe (binária) }\end{array}$ & 0.0055383 & 0.005 & 1.218 & 0.223 \\
\hline $\begin{array}{l}\text { Superior completo ou incompleto } \\
\text { do pai (binária) }\end{array}$ & -0.0011777 & 0.005 & -0.261 & 0.794 \\
\hline Tempo de conclusão do $2^{\circ}$ grau & 0.0007677 & 0.000 & 1.695 & 0.090 \\
\hline
\end{tabular}

Fonte: Cálculos do autor a partir da PED-RMS e base de dados do Vestibular/UFBa. A amostra foi composta de $n=6.440$ casos. 
Como em todas as estimativas, a renda vitalícia mostrou-se altamente significativa, porém, o custo de oportunidade assim não se mostrou, nem ao nível de significância de 64\%. O intercepto correspondendo a um valor de 0,002, foi bastante significativo; este intercepto pode ser interpretado como a fração da renda vitalícia que é destinada à educação superior. Outra variável que não se mostrou significativa foi a renda familiar, provavelmente porque a grande maioria dos alunos que ingressam na universidade são provenientes de famílias que têm uma renda familiar elevada. Todos os cursos mostraram-se estatisticamente significativos, ao nível de $0 \%$, praticamente.

Outras variáveis que não se mostraram significativas foram: se o indivíduo é ou não da capital, o sexo do indivíduo, se é solteiro ou não; além destes, não se mostraram significativos, contrariando a literatura na área, foi o fato de o pai ou a mãe terem o curso superior completo ou incompleto versus ter uma escolaridade média ou inferior, e o tempo de conclusão do curso de $2^{\circ}$. grau. $\mathrm{O}$ modelo, entretanto, mostrou-se com um poder de explicação excepcionalmente elevado, de $98 \%$, revelando a sua forte aderência aos fatos.

A variável que mais contribui para esta explicação é, de longe, a renda vitalícia, a despeito de o questionário socioeconômico respondido pelo candidato no ato da inscrição revelar que apenas 1,4\% atribuem às perspectivas de renda proporcionadas pela profissão como sendo relevante na decisão.

\subsection{Estática Comparativa}

A estática comparativa é uma técnica que permite captar o efeito de variáveis exógenas ou parâmetros sobre as variáveis endógenas. Neste caso, procurar-se-á captar o efeito da taxa de juro sobre a demanda, da duração do curso e da experiência no mercado de trabalho do candidato.

Para isto, serão substituídas as equações (11) e (15) na equação (6) e serão calculadas as derivadas parciais relacionadas a cada uma das variáveis exógenas mencionadas.

Os resultados encontrados são os seguintes:

$$
\left(\partial \mathrm{c}_{\mathrm{j}}^{\mathrm{d}} / \partial \beta\right)<0
$$

Este resultado da equação (33) informa que um aumento da taxa de juro reduz a demanda por ensino superior. Sucede que tanto o fluxo de 
renda vitalícia quanto o fluxo do custo de oportunidade se reduzem, mas o efeito sobre a renda vitalícia é maior, em decorrência desta se referir a um período de tempo mais longo e o sinal da variável preço é negativo; prepondera, entretanto, o "efeito renda" sobre o "efeito preço", reduzindose a demanda. Utilizou-se para este cálculo a taxa de juro instantânea de $11,3329 \%$ ao ano, equivalente a $12 \%$ ao ano, em capitalização descontínua.

$$
\left(\partial c_{j} d / \partial D\right)<0
$$

A equação 34 vem confirmar a intuição: quanto mais longa a duração do curso, menor a demanda, pois maiores são os custos de oportunidade.

Um resultado menos óbvio é o da derivada da equação 35:

$$
\left(\partial c_{j}{ }^{d} / \partial E\right)>0
$$

Esta derivada informa que quanto maior a experiência do candidato no mercado de trabalho maior a demanda por educação; isto demonstra a complementaridade entre educação e mercado de trabalho, uma vez que as exigências do mercado de trabalho levam as pessoas a se atualizarem de modo a reduzir e/ou retardar a data de início da depreciação do seu estoque de capital humano. Este resultado decorre da constatação de que a experiência aumenta a renda vitalícia das pessoas, refletindo-se em uma maior demanda por ensino superior.

\subsection{A Isodemanda}

Um último exercício, mas nem por isso menos interessante, é o que permite calcular a taxa marginal de substituição entre o custo de oportunidade do aluno e a sua renda vitalícia, ambas calculadas no ponto médio. Dada a função geral de demanda com os argumentos preço e renda vitalícia simplesmente:

$$
c_{j}{ }_{j}=c_{j}\left(p_{j}, R_{j}\right)
$$


Derivando-se totalmente (36), fazendo-se $\mathrm{dc}_{\mathrm{j}}=0$, re-arrumando os termos e tomando-se a equação estimada (21) para cálculo desta taxa de substituição, tem-se:

$$
\left(\mathrm{dR}_{\mathrm{j}} / \mathrm{d} \mathrm{p}_{\mathrm{j}}\right)=\left(-\partial \mathrm{c}_{\mathrm{j}} / \partial \mathrm{p}_{\mathrm{j}}\right) /\left(\partial \mathrm{c}_{\mathrm{j}} / \partial \mathrm{R}_{\mathrm{j}}^{\mathrm{l}}\right)=0,2517289073 .\left(\mathrm{R}_{\mathrm{j}} / \mathrm{p}_{\mathrm{j}}\right)
$$

A equação (37) é o simétrico do efeito-preço dividido pelo efeitorenda vitalícia. Ela pode ser interpretada como uma função isodemanda, porque ela mostra quanto o indivíduo terá que incrementar a sua renda vitalícia se o seu custo de oportunidade se eleva, de modo a permanecer na mesma curva de demanda.

Tomando-se os preços e renda vitalícia médios, resulta em:

$$
\left(\mathrm{dR}_{\mathrm{j}} / \mathrm{dp}_{\mathrm{j}}\right)=2,78
$$

Ou seja, segundo (38), o indivíduo médio está disposto a sacrificar $\mathrm{R} \$ 1,00$ para obter um incremento de $\mathrm{R} \$ 2,78$ a título de renda vitalícia.

\section{CONCLUSÕES}

Resultados importantes foram obtidos neste artigo, como, por exemplo, verificar que a decisão de fazer ou não fazer o curso superior é fortemente influenciada pela perspectiva de renda futura que o candidato tem, sem desconsiderar os custos de renda sacrificada que ele incorrerá durante o curso.

Muitos resultados não se mostraram significativos, acredita-se, por ter sido utilizada a base de dados relativa apenas aos aprovados, após, portanto, terem passado pelo filtro do Vestibular. Muito provavelmente estas variáveis se mostrarão significativas estatisticamente, em próximo trabalho em que se utilize a base de todos os candidatos inscritos.

Um outro senão é que a presente modelagem não permite identificar as variáveis que afetam a escolha de cada curso em particular, o que, acredita-se, poderá ser revelado por meio da técnica de regressão logística. Mas a abordagem aqui utilizada, por outro lado, permite a realização de certos cálculos que não seriam possíveis pela abordagem alternativa. São exemplos disso, os cálculos das elasticidades-preço e renda 
e da taxa marginal de substituição entre custo de oportunidade e renda vitalícia. Essa taxa, inclusive, poderia ser calculada para cada curso.

Além dos modelos terem um alto poder de explicação, chegando a $90 \%$ com apenas duas variáveis, e a $98 \%$ na regressão da Tabela 1, os resultados demonstram que a demanda por ensino superior público e gratuito é muito pouco preço-elástica, mas é um bem sensível a variações de renda vitalícia.

O exercício de estática comparativa também revelou três coisas interessantes: quanto maior a taxa real de juro, menor a demanda; quanto maior a experiência do aluno no mercado de trabalho, maior a demanda, e, terceiro, e já esperado, de que, quanto maior a duração do curso, menor a demanda pelo ensino superior. Esse exercício de estática comparativa é ainda mais relevante quando se considera o poder de explicação dos modelos de demanda utilizados.

Ademais, os cursos da área 1, por exemplo, possuem uma menor demanda, provavelmente em decorrência da maior duração do curso, acarretando maiores custos de oportunidade, e em decorrência da maior taxa de desemprego para a profissão de engenharia civil, principalmente.

Quanto à questão étnica e de sexo, estas variáveis não se mostram significativas, acredita-se, por causa da base de dados utilizada, o que poderia lançar luzes sobre o debate da necessidade de existência ou não de cotas para negros e mulheres.

\section{REFERÊNCIAS BIBLIOGRÁFICAS}

AVENA, C.P. Os Retornos do Capital Humano na Região Metropolitana de Salvador. Salvador, 2000. Dissertação (mestr.) Universidade Federal da Bahia / Curso de Mestrado em Economia, 116p.

CARRERA-FERNANDEZ, J. Microeconomia. CME/UFBa, 1998. (mimeo)

DESJARDINS, S.; DUNDAR, H.; HENDEL, D. Modeling the College Application Decision Process in a Land Grant University. Economics of Education Review, n.18, 1999. p.117-132.

FREEMAN, C. Education and Technological Change. Cambridge Journal of Economics, v. 43, n. 1, October 1995.

HAGY, A.; STANIEC, J.F.O. Immigrant status, race, and institutional choice in higher education. Economics of Education Review, n. 21, 2002. p.381392. 
HOSSLER, D.; BRAXTON, J.; COOPERSMITH, G. Understanding Student College Choice. In: SMART, J. (ed.). Higher Education Handbook of Theory and Research, v. V, New York: Agathon Press, 1989. p. 231-288.

KREPS, D.M. A Course in Microeconomic Theory. New Jersey: Princeton University Press, 1990. 839p.

MCFADDEN, D. Conditional Logit Analysis of Qualitative Choice Behavior. In: ZAREMBKA, P. (ed.). Frontier in Econometrics. New York: Academic Press, 1973, p.105-142.

MAS-COLELL,A.; WHINSTON, M.D.; GREEN, J.R. Microeconomic Theory. Oxford: Oxford University Press, 1995, 981p.

MINCER, J. Schooling, Experience and Earnings. New York: NBER, 1974, 206p.

MONTMARQUETTE, C.; CANNINGS, K.; MAHSEREDJIAN, S. How do young people choose college majors? Economics of Education Review, n.21, 2002, p.543-556.

GREEN, W.H. Econometric Analysis. 4. ed. New Jersey: Prentice Hall, 2000, 1004p.

RADNER, R.; MILLER, L.S. Demand and Supply in U.S. Higher Education: A Progress Report. American Economic Review.

STIGLITZ, J. The Demand for Education in Public and Private School Systems. Journal of Public Economics, n.3, 1974, p.349-385.

TOBIAS, J. Model uncertainty and race and gender heterogeneity in the college entry decision. Economics of Education Review, n. 21, 2002, p.211-219.

VARIAN, H. R. Microeconomic Analysis, 2.ed. Ed. New York: W.W. Norton \& Co, 1984, 350p.

VERHINE, R.E. Ensino Superior no Brasil: Características e Desafios, 2002. (mimeo)

Recebido em: outubro 2003 Aprovado para publicação em: fevereiro 2004 